\title{
NONLINEAR PERTURBATION OF $m$-ACCRETIVE OPERATORS
}

\author{
W. E. FITZGIBBON ${ }^{1}$
}

\begin{abstract}
Let $X$ be a reflexive Banach space. Conditions sufficient to guarantee that the sum $A+B$, of two $m$-accretive operators $A$ and $B$ is $m$-accretive are provided. The basic requirements are that the operator $B$ be bounded in some sense relative to $A$ and that $A$ and $B$ be weakly closed.
\end{abstract}

The object of this paper is the investigation of the additive perturbation of a $m$-accretive, possibly nonlinear, operator $A$ by a $m$-accretive, possibly nonlinear, operator $B$. Our basic requirements are that the Banach space be reflexive and that the operator $B$ be bounded in some sense relative to $A$. Our results relate to the work of T. Kato [8] and J. Mermin [12] concerning perturbation in Banach spaces which have uniformly convex dual. For recent work concerning perturbation the reader is referred to G. F. Webb [14], [15], V. Barbu [1], J. Goldstein [6] and Y. Konishi [11].

In what follows $X$ will be a reflexive Banach space with norm $\|\cdot\|$; $X^{*}$ will denote the dual space of $X$, and the pairing between $X$ and $X^{*}$ will be denoted by $\langle$,$\rangle . If A$ and $B$ are operators mapping subsets of $X$ to $X$ we define the sum $A+B$ by the equation $(A+B) x=A x+B x$ for $x \in D(A) \cap D(B)$.

DEFINITION 1.1. Let $A$ be a nonlinear operator mapping a subset of a Banach space $X$ to $X$. $A$ is said to be accretive provided that $\| x+\lambda A x-$ $(y+\lambda A y)\|\geqq\| x-y \|$ for all $\lambda \geqq 0$ and $x, y \in D(A)$. An accretive operator is said to be $m$-accretive provided that $R(I+\lambda A)=X$ for all $\lambda \geqq 0$.

T. Kato [9] has shown that the definition of accretiveness is equivalent to the statement that $\operatorname{Re}\langle A x-A y, f\rangle \geqq 0$ for $x, y \in D(A)$ and some $f \in F(x-y)$ where $F$ is the duality map from $X$ to $X^{*}$. If $A$ is an $m$-accretive operator, $A$ has no proper accretive extension. However not every maximal accretive operator is $m$-accretive. If $A$ is accretive and $n \in Z^{+}$we define

Presented to the Society, November 17, 1973; received by the editors June 4, 1973 and, in revised form, September 28, 1973.

AMS (MOS) subject classifications (1970). Primary 47H15, 34G05; Secondary 47B44, 47D05.

Key words and phrases. Accretive, $m$-accretive weakly closed, perturbation of type $K$, nonlinear operator.

${ }^{1}$ Supported in part by the University of Houston RIP Grant.

(c) American Mathematical Society 1974 
$J_{n} x=(I+1 / n A)^{-1} x$ for $x \in D_{n}=R(I+1 / n A)$. We define the Yosida approximations $A_{n} x=n\left(I-J_{n}\right) x$. The following facts are well known:

$$
\begin{aligned}
\left\|J_{n} x-J_{n} y\right\| & \leqq\|x-y\| & & \text { for } x, y \in D_{n}, \\
\left\|J_{n} x-x\right\| & \leqq n^{-1}\|A x\| & & \text { for } x \in D(A) \cap D_{n}, \\
A_{n} x & =A J_{n} x & & \text { for } x \in D_{n}, \\
\left\|A_{n} x\right\| & \leqq\|A x\| & & \text { for } x \in D(A) \cap D_{n} .
\end{aligned}
$$

If $A$ is an $m$-accretive operator then $A_{n}$ is an everywhere defined Lipschitz continuous accretive operator with Lipschitz constant $2 n$.

Henceforth we shall use the symbol " $\rightarrow$ " to denote strong convergence in $X$ and " $\rightarrow$ " to denote weak convergence in $X$.

Definition 1.2. Let $A$ be a nonlinear operator mapping a subset of $X$ to $X . A$ is said to be weakly closed provided that $\left\{x_{n}\right\} \subseteq D(A), x_{n} \rightarrow x$, and $A x_{n} \rightarrow y$ imply that $x \in D(A)$ and $A x=y$.

If $X$ is a reflexive Banach space and $A$ is a weakly closed operator then $A$ has the following property: if $\left\{x_{n}\right\} \subseteq D(A), x_{n} \rightarrow x$ and $\left\|A x_{n}\right\| \leqq M$ for some $M>0$ then $A x_{n} \rightarrow A x$. Let us refer to this property of operators as condition $W$. We now specify the type of perturbation to be considered.

Definition 1.3. Let $A$ and $B$ be nonlinear operators defined on subsets of a Banach space $X$. If $D(A) \subseteq D(B)$ then $B$ is said to be a perturbation of $A$ of type $K$ provided that there exist constants $a<1, b \geqq 0$ and $c \geqq 0$ so that,

$$
\|B x\| \leqq a\|A x\|+b\|x\|+c \text { for } x \in D(A) .
$$

We now make precise our notion of strong solutions to the Cauchy initial value problem.

Definition 1.4. Let $A$ be an operator defined on a subset of a Banach space $X$. By a strong solution to the Cauchy initial value problem,

$$
u^{\prime}(t)+A u(t)=0 ; \quad u(0)=x, t \in[0, T),
$$

we mean a function $u:[0, T) \rightarrow X$ such that $u$ is Lipschitz continuous on compact subsets of $[0, T) ; u(0)=x ; u^{\prime}(t)$ exists and satisfies (1.3) for a.e. $t \in[0, T)$.

We now provide conditions sufficient for the sum of two operators to be accretive.

LemMA 1.1. Let $A$ and $B$ be nonlinear accretive operators on a Banach space $X$. If $B$ is m-accretive and satisfies condition $W$ then $A+B$ is accretive.

Proof. Let $x \in D(B)$ then condition $W$ and equations (1.1) imply that $B_{n} x=n\left(I-(I+1 / n B)^{-1}\right) x$ converges weakly to $B x$. Since $B$ is $m-$ accretive, $B_{n}$ is accretive and everywhere defined. Moreover we can make use of the equivalent formulation of accretiveness to observe that $B_{n}$ 
is strongly accretive, i.e. $\operatorname{Re}\left\langle B_{n} x-B_{n} y, f\right\rangle \geqq 0$ for all $f \in F(x-y)$. The accretiveness of $A$ implies that for each $x, y \in D(A)$ there is an $f^{\prime} \in F(x-y)$ so that $\left\langle A x-A y, f^{\prime}\right\rangle \geqq 0$. If we assume that $x, y \in D(A) \cap D(B)$ we can conclude that

$$
\operatorname{Re}\left\langle(A+B x)-(A+B) y, f^{\prime}\right\rangle=\lim \left\langle\left(A+B_{n}\right) x-\left(A+B_{n}\right) y, f^{\prime}\right\rangle \geqq 0 .
$$

We now proceed to establish a global existence theorem for Cauchy problems involving the operator $A+B$.

THEOREM 1. Let $A$ and $B$ be weakly closed m-accretive operators defined on a reflexive Banach space $X$. If $B$ is a perturbation of type $K$ of $A$ then for each $x \in D(A)$ there exists a unique global solution to the Cauchy initial value problem

$$
u^{\prime}(t)+(A+B) u(t)=0 ; \quad u(0)=x \text { for a.e. } t \in[0, \infty) .
$$

Proof. We proceed by picking an arbitrary $T<\infty$ and demonstrating that (1.4) has a unique strong solution on $[0, T]$. Denoting $B_{n} x=$ $n\left(I-(I+1 / n B)^{-1}\right) x$, we consider the operators $A+B_{n}$. A recent result of V. Barbu [1] together with the $m$-accretiveness of $A$ and the continuity of $B_{n}$ insures that $A+B_{n}$ is $m$-accretive. We now consider the approximate Cauchy problems

$$
u_{n}^{\prime}(t)+\left(A+B_{n}\right) u_{n}(t)=0 ; \quad u(0)=x \quad \text { for } t \in[0, T] .
$$

The $m$-accretiveness of $A+B_{n}$ together with recent results of Crandall and Liggett [3] and Brezis and Pazy [2] guarantee that for each $n \in Z^{+}$, (1.5) has a strong solution; moreover each $u_{n}(t)$ may be represented as the product integral $u_{n}(t)=\lim _{m \rightarrow \infty}\left(I+t\left(A+B_{n}\right) / m\right)_{x}^{-m}$ uniformly for $t \in$ $[0, T]$. Repeated applications of the fourth assertion of (1.1) yield

$$
\left\|\left(A+B_{n}\right)\left(I+t\left(A+B_{n} n\right) / m\right)_{x}^{-m}\right\| \leqq\|A x\|+\|B x\| .
$$

We observe that condition $W$ implies that $\left(A+B_{n}\right)\left(I+t\left(A+B_{n}\right) / m\right)_{x}^{-m} \rightarrow$ $\left(A+B_{n}\right) u_{n}(t)$ and thereby insures the existence of a constant $M>0$ so that

$$
\begin{gathered}
\left\|u_{n}(t)-u_{n}(\tau)\right\| \leqq|t-\tau| M \quad \text { for } t, \tau \in[0, T] \\
\left\|u_{n}^{\prime}(t)\right\|=\left\|\left(A+B_{n}\right) u_{n}(t)\right\| \leqq M \quad \text { for a.e. } t \in[0, T]
\end{gathered}
$$

We now claim that there is a subsequence $\left\{u_{n^{\prime}}(t)\right\}$ of $\left\{u_{n}(t)\right\}$ which converges weakly to a function $u(t)$ which also satisfies the Lipschitz condition (1.6) with constant $M$. The argument of Lemma 2.1 [5] is directly applicable to establish this convergence. We relabel the weakly convergent subsequence as $\left\{u_{n}(t)\right\}$.

We now seek to ascertain that $\left(A+B_{n}\right) u_{n}(t) \rightarrow(A+B) u(t)$. Since $u_{n}(t) \in D(A), u_{n}(t) \in D(B)$; using properties of perturbations of type $K$ 
and statements (1.1) and (1.6) we have

$$
\begin{aligned}
(1-a)\left\|A u_{n}(t)\right\|-b\left\|u_{n}(t)\right\|-c & \leqq\left\|A u_{n}(t)\right\|-\left\|B u_{n}(t)\right\| \\
& \leqq\left\|A u_{n}(t)\right\|-\left\|B_{n} u_{n}(t)\right\| \leqq M .
\end{aligned}
$$

Because $a<1$ we can conclude that there is a constant $M^{\prime}$ so that

$$
\begin{aligned}
\left\|A u_{n}(t)\right\| & \leqq M^{\prime} \quad \text { for } t \in[0, T], \\
\left\|B_{n} u_{n}(t)\right\| \leqq M^{\prime} & \text { for } t \in[0, T] .
\end{aligned}
$$

That $A u_{n}(t) \rightarrow A u(t)$ follows from the fact that weakly closed operators satisfy condition $W$. To see that $B_{n} u_{n}(t) \rightarrow B u(t)$ we recall that $B_{n} x=$ $B\left(I+n^{-1} B\right)_{x}^{-1}$; use statement $(1.1)$ to see that $\left(I+n^{-1} B\right)^{-1} u_{n}(t) \rightarrow u(t)$ and apply condition $W$.

Since $u_{n}(t)$ is a strong solution to (1.5) we have the following equation:

$$
\begin{aligned}
& \left\langle u_{n}(t), f\right\rangle=\langle x, f\rangle-\int_{0}^{t}\left\langle\left(A+B_{n}\right) u_{n}(s), f\right\rangle d s \\
& \qquad \text { for } x \in D(A), f \in X^{*}, t \in[0, T] .
\end{aligned}
$$

Taking the limit as $n \rightarrow \infty$ of each side of (1.9) we obtain $\langle u(t), f\rangle=\langle x, f\rangle-$ $\int_{0}^{t}\langle(A+B) u(s), f\rangle d s$, and hence deduce that

$$
u(t)=x-\int_{0}^{t}(A+B) u(s) d s \quad \text { for } t \in[0, T] .
$$

The above integral can be differentiated and we obtain $u^{\prime}(t)+$ $(A+B) u(t)=0$ for a.e. $t \in[0, T]$ and $u(0)=x$. The uniqueness of solutions to (1.4) on $[0, T]$ follows from the accretiveness of $A+B$ and standard techniques, cf. [9].

The next lemma connects the $m$-accretiveness of an accretive operator with the existence of strong solutions to a Cauchy initial value problem, cf. Kato [8].

Lemma 1.2. Let $X$ be a Banach space and $A$ be a closed nonlinear accretive operator. Then $A$ is m-accretive provided that there exists an $x \in D(A)$ such that for all $p \in X$ the Cauchy initial value problem

$$
u^{\prime}(t)+(A+I) u(t)-p=0 ; \quad u(0)=x, \quad t \in[0, \infty),
$$

has a strong solution.

Proof. Let $x \in D(A)$ satisfy the hypothesis of the lemma; we shall use the existence of a solution to (1.10) to show that there is a $v \in D(A)$ so that $v=\lim _{t \rightarrow \infty} u(t)$ and $(A+I) v-p=0$. Thus $R(I+A)=X$ and we can refer to a result of Oharu [13] to guarantee that $R(I+\lambda A)=X$ for all $\lambda \geqq 0$. 
Utilizing the accretiveness of $A$ and standard techniques we see that for a.e. $t, h \in[0, \infty)$ and $f \in F(u(t+h)-u(t))$,

$$
\begin{aligned}
(d / d t)\|u(t+h)-u(t)\|^{2} & =-2 \operatorname{Re}\langle(A+I) u(t+h)-(A+I) u(t), f\rangle \\
& \leqq-2\|u(t+h)-u(t)\|^{2}
\end{aligned}
$$

and hence that

$$
(d / d t)\left\{e^{2 t}\|u(t+h)-u(t)\|^{2}\right\} \leqq 0 .
$$

We integrate $(2.11)$ on $(0, t)$ to obtain the inequality

$$
\|u(t+h)-u(t)\| \leqq e^{-t}\|u(h)-u(0)\| .
$$

Since $u$ is a strong solution to the Cauchy problem we can conclude that there is a $M>0$ so that

$$
\left\|u^{\prime}(t)\right\| \leqq e^{-t} M \quad \text { for a.e. } t \in[0, \infty)
$$

Since $\|u(t+h)-u(t)\| \leqq \int_{t}^{t+h}\left\|u^{\prime}(s)\right\| d s$ we use (2.12) to conclude that there is a $v=\lim _{t \rightarrow \infty} u(t)$. Let $\left\{t_{i}\right\}_{i=1}^{\infty}$ be an increasing sequence of numbers at which equation (1.10) is satisfied such that $\lim t_{i}=\infty$. By virtue of (2.12) we have $\lim \left\|(A+I) u\left(t_{i}\right)-p\right\|=0$ and thus we can invoke the closedness of $A$ to establish $(A+I) v=p$.

We are now in a position to state and prove our principal result.

Theorem 2. Let $X$ be a reflexive Banach space and let $A$ and $B$ be nonlinear, weakly closed m-accretive operators such that $D(A) \subseteq D(B)$. If $B$ is a perturbation of $A$ of type $K$ then $A+B$ is $m$-accretice.

Proof. Theorem 2 is obtained by defining $B_{p}, x=(B+I) x-p$. Clearly if $B$ satisfies the hypotheses of Theorems 1 and 2 then so does $B_{p}$. Thus Theorem 2 follows from Theorem 1 by immediate application of Lemma 1.2.

If $A$ is linear we have the following corollary:

COROllary. Let $A$ be a closed linear m-accretice operator defined on a reflexive Banach space $X$. If $B$ is a nonlinear, neakly closed, m-accretive perturbation of $A$ of type $K$, then $A+B$ is m-accretive.

Proof. We need only observe that a strongly closed linear operator is weakly closed.

If we further require that the operator $B$ be everywhere defined and weakly continuous, i.e., that $B$ map weakly convergent sequences to weakly convergent sequences, we can eliminate the requirement that $B$ be bounded relative to $A$.

THEOREM 3. Let $A$ be a closed, linear m-accretive operator on a reflexive Banach space. If $B$ is a nonlinear, leakly continuous accretive operator then $A+B$ is m-accretive. 
Proof. In [4] it has been shown that a weakly continuous accretive operator on a reflexive Banach space is $m$-accretive. Following the proof of Theorem 1 we guarantee the existence of unique solutions to the approximate equations $u_{n}^{\prime}(t)+\left(A+B_{n}\right) u_{n}(t)=0 ; u_{n}(0)=x, t \in[0, T]$.

As before we obtain the existence of $M>0$ so that $\left\|u_{n}(t)-u_{n}(\tau)\right\| \leqq$ $|t-\tau| M$ and $\left\|\left(A+B_{n}\right) u_{n}(t)\right\| \leqq M$. We now observe that weakly continuous operators in reflexive spaces map bounded subsets to bounded subsets. Since $\left\|\left(I+n^{-1} B\right)^{-1} u_{n}(t)-u_{n}(t)\right\| \leqq n^{-1}\left\|B u_{n}(t)\right\|$ and $\left\|B_{n} u_{n}(t)\right\| \leqq$ $\left\|B u_{n}(t)\right\|$ we see that $\left\|B_{n} u_{n}(t)\right\|$ and $\left\|A u_{n}(t)\right\|$ are bounded independently of $n$. We are now able to apply the remainder of the previous argument.

\section{REFERENCES}

1. V. Barbu, Continuous perturbations of nonlinear m-accretive operators in Banach spaces, Boll. Un. Mat. Ital. 6 (1972), 270-278.

2. H. Brezis and A. Pazy, Accretive sets and differential equations in Banach spaces, Israel J. Math. 8 (1970), 367-383. MR 43 \#1000.

3. M. Crandall and T. Liggett, Generation of semigroups of nonlinear transformations on general Banach spaces, Amer. J. Math. 93 (1971), 265-298. MR 44 \#4563.

4. W. Fitzgibbon, Weakly continuous accretive operators, Bull. Amer. Math. Soc. 2 (1973), 473-474.

5. - Weakly continuous accretive operators in reflexive Banach spaces, Proc. Amer. Math. Soc. 41 (1973), 229-236.

6. J. Goldstein, A perturbation theorem for evolution equations and applications, Illinois J. Math (to appear).

7. E. Hille and R. S. Phillips, Functional analysis and semi-groups, rev. ed., Amer. Math. Soc. Colloq. Publ., vol. 31, Amer. Math. Soc., Providence, R.I., 1957. MR 19, 664.

8. T. Kato, Accretive operators and nonlinear evolution equations in Banach spaces, Proc. Sympos. Pure Math., vol. 18, Part 1, Amer. Math. Soc., Providence, R.I., 1970, pp. 138-161. MR 42 \#6663.

9. - Nonlinear semigroups and evolution equations, J. Math. Soc. Japan 19 (1967), 508-520. MR 37 \#1820.

10. — Perturbation theory for linear operators, Die Grundlehren der math. Wissenschaften, Band 132, Springer-Verlag, New York, 1966. MR 34 \#3324.

11. Y. Konishi, A remark on perturbation of m-accretive operators in Banach spaces, Proc. Japan Acad. 45 (1969), 850-853.

12. J. Mermin, Accretive operators and nonlinear semigroups, Thesis, University of California, Berkeley, Calif., 1968.

13. S. Ôharu, Note on the representation of semigroups of nonlinear operators, Proc. Japan Acad. 42 (1966), 1149-1154. MR 36 \#3167.

14. G. F. Webb, Continuous nonlinear perturbations of nonlinear operators in Banach spaces, J. Functional Analysis 10 (1972), 191-203.

15. - Nonlinear perturbations of linear accretive operators in Banach spaces, Israel J. Math. 12 (1972), 237-248.

16. C. Yen, On the sum of two nonlinear m-dissipative sets (to appear).

Department of Mathematics, University of Houston, Houston, Texas 77004 